\title{
Growth and characterization of hexamethylenetetramine crystals grown from solution
}

\author{
B. BABU, J. CHANDRASEKARAN*, S. BALAPRABHAKARAN \\ Department of Physics, Sri Ramakrishna Mission Vidyalaya College of Arts and Science, \\ Coimbatore - 641 020, Tamil Nadu, India
}

\begin{abstract}
Organic nonlinear optical single crystals of hexamethylenetetramine (HMT; $10 \times 10 \times 5 \mathrm{~mm}^{3}$ ) were prepared by crystallization from methanol solution. The grown crystals were subjected to various characterization techniques such as single crystal XRD, powder XRD, UV-Vis and electrical studies. Single crystal XRD analysis confirmed the crystalline structure of the grown crystals. Their crystalline nature was also confirmed by powder XRD technique. The optical transmittance property was identified from UV-Vis spectrum. Dielectric measurements were performed as a function of frequency at different temperatures. DC conductivity and photoconductivity studies were also carried out for the crystal. The powder second harmonic generation efficiency (SHG) of the crystal was measured using Nd:YAG laser and the efficiency was found to be two times greater than that of potassium dihydrogen phosphate (KDP).
\end{abstract}

Keywords: hexamethylenetetramine; crystal growth; nonlinear optical material (NLO); dielectrics

(C) Wroclaw University of Technology.

\section{Introduction}

The rapid development of optical communication systems has led to the search for new materials with remarkable nonlinear optical properties which are used in the realization of signal processing devices. Optical techniques for modulating, amplifying, transforming signals and information can be faster than electrical techniques. Their use could allow the solution of a range of problems which arise in integrated electronic circuits and electronic telecommunications networks. The highly conjugated organic compounds are among the materials having high nonlinear optical coefficients $[1,2]$. In NLO materials, organic compounds have been investigated for their potentially high nonlinearity and electro-optic response compared with inorganic materials. The advantages offered by the organic over inorganic systems include high electric susceptibility.

Hexamethylenetetramine known as urotropine or tetraazaadamantane is one of the exceptional compounds that exhibits nonlinear optical prop-

*E-mail: jchandaravind@yahoo.com erties and crystallizes in the cubic system. It is used for the growth of $\mathrm{ZnO}$ nanorods and in cyclotrimethylenetrinitramine (RDX) production [311]. Some authors have already tried to grow the defect free crystals from solutions by different methods. In the present work, optically good quality single crystals of hexamethylenetetramine were grown by slow evaporation solution growth technique at a constant temperature of $35{ }^{\circ} \mathrm{C}$. The grown crystals were characterized by single crystal X-ray diffraction analysis, optical transmittance, electrical analysis, microhardness test and powder SHG analysis.

\section{Experimental procedures}

\subsection{Crystal growth}

Organic materials that are commercially available have unpredictable quality, with purity in the range of $95-99 \%$. The most essential feature to grow high quality crystals is the purity of the material and the solution. The commercially available hexamethylenetetramine (Merck, $99 \%$ ) was purified by repeated recrystallization from methanol. Large single crystals of hexamethylenetetramine 


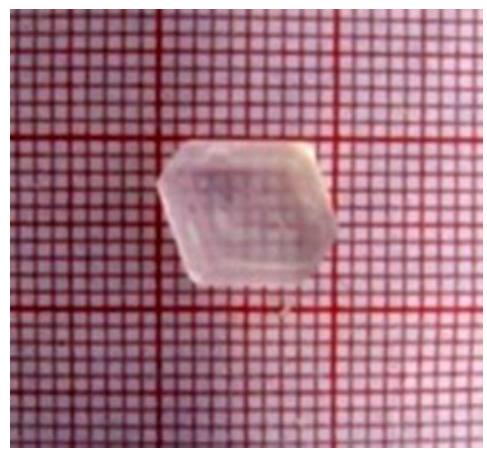

Fig. 1. As grown hexamethylenetetramine single crystal.

were grown by the slow evaporation technique at a constant temperature of $35^{\circ} \mathrm{C}$. The saturated solution was poured into a crystal vessel and covered with a perforated lid to reduce the evaporation of the solvent. Spontaneously nucleated crystals were obtained at the size of $\left(10 \times 10 \times 5 \mathrm{~mm}^{3}\right)$ at a time interval of 10 days. Fig. 1 depicts the photograph of an as grown crystal of HMT.

\section{Results and discussion}

\section{1. $\mathrm{X}$-ray diffraction analysis}

In order to acquire the crystal data, the grown crystals were subjected to single crystal X-ray studies using ENRAF NONIUS CAD-4 X-ray diffaractometer with $\operatorname{Mo~} \mathrm{K} \alpha(\lambda=0.7107 \AA)$ radiation. The crystallographic data reveal that the grown crystal belongs to the cubic system with noncentrosymmetric space group $\mathrm{I} 43 \mathrm{~m}$ and lattice parameters $a=6.082 \AA, b=6.082 \AA, c=6.082 \AA$, $V=173.567 \AA^{3}[12]$. The calculated values of cell parameters are in good agreement with the earlier reported values, which are given in Table 1 . The grown crystal was subjected to powder XRD analysis and the spectrum is shown in Fig. 2. The powder XRD pattern of the crystal was recorded with a RICH SIEFERT X-ray powder diffractometer using $\mathrm{Cu} \mathrm{K} \alpha(\lambda=1.5405 \AA)$ radiation. The sample was scanned in the $2 \theta$ range of $5-80^{\circ}$ at a scan rate of $1 \% \mathrm{~min}$. The sharp and well defined Bragg' peaks at specific range of $2 \theta$ angles confirm the crystalline nature and purity of the crystal.

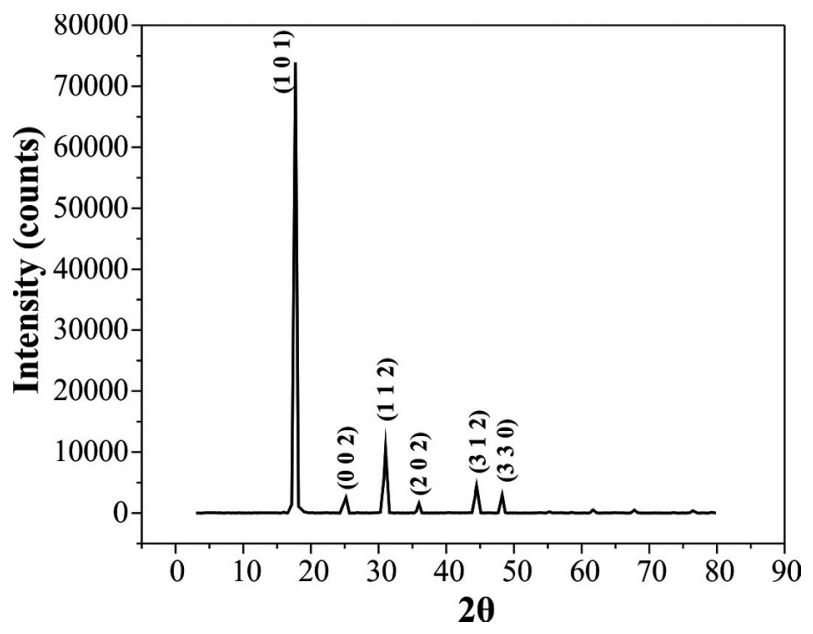

Fig. 2. Powder XRD pattern of HMT crystal.

Table 1. Crystallographic data for HMT.

\begin{tabular}{|c|c|c|}
\hline Data & Ref [12] & Present work \\
\hline Formula & $\mathrm{C}_{6} \mathrm{H}_{12} \mathrm{~N}_{4}$ & $\mathrm{C}_{6} \mathrm{H}_{12} \mathrm{~N}_{4}$ \\
\hline Crystal system & Cubic & Cubic \\
\hline$a(\AA)$ & 6.082 & 6.080 \\
\hline$b(\AA)$ & 6.084 & 6.080 \\
\hline$c(\AA)$ & 6.088 & 6.080 \\
\hline$\alpha\left(^{\circ}\right)$ & 109.35 & 109.47 \\
\hline$\beta\left(^{\circ}\right)$ & 109.47 & 109.47 \\
\hline$\gamma\left({ }^{\circ}\right)$ & 109.47 & 109.47 \\
\hline$V\left(\AA^{3}\right)$ & 173.567 & 173.048 \\
\hline Space group & $\mathrm{I} 43 \mathrm{~m}$ & $\mathrm{I} 43 \mathrm{~m}$ \\
\hline$Z$ & 2 & 2 \\
\hline
\end{tabular}

\subsection{UV-Vis transmittance studies}

The UV-Vis spectrum was recorded using a Perkin-Elmer Lambda 35 spectrometer in the range of $200-1100 \mathrm{~nm}$ covering the near ultraviolet $(200-400 \mathrm{~nm})$, visible $(400-800 \mathrm{~nm})$ and then far infrared region $(800-1200 \mathrm{~nm})$ to find the suitability of the grown crystal for optical applications. The cut-off wavelength of HMT crystal is $237 \mathrm{~nm}$ and the percentage of transmission was found to be $85 \%$. The large transmittance window and absence of absorption in the visible region is an advantage as it is the essential condition for materials having NLO properties [13]. 


\subsection{Dielectric studies}

Generally, dielectric studies provide useful information about electric field distribution and charge transport mechanism in a crystal, the nature of atoms, chemical bondings and their polarization mechanism. A good quality single crystal with proper faces, with the size of $7 \times 7 \times 3 \mathrm{~mm}^{3}$ was chosen for the dielectric measurement. The crystal was polished and electronic grade silver paste was applied to the opposite sides of the crystal to make a capacitor with the crystal as a dielectric medium. The dielectric studies were carried out using a HIOKI 3532-50 LCR HITESTER impedance meter. The experiment was carried out at different frequencies from $50 \mathrm{~Hz}$ to $5 \mathrm{MHz}$ at various temperatures ( 303 to $328 \mathrm{~K}$ ). The dielectric constant was calculated using the relation:

$$
\varepsilon_{r}=\frac{C d}{\varepsilon_{0} A}
$$

where $\mathrm{C}$ is the capacitance and $\mathrm{d}$ is the thickness, $A$ is the cross sectional area of the sample and $\varepsilon_{0}$ is the free space permitivity of the sample. From Fig. 3 and Fig. 4 it can be observed that dieletric constant is very high at low frequency then decreases slowly and attains a constant value at higher frequencies. Very high dielectric constant at low frequency may be due to the presence of all four polarization modes, namely electronic, ionic, orientational and space charge polarization [14]. At a very high frequency vibration may occur, which causes that the dipoles are not able to follow the alternating field and the dielectric constant decreases with an increase in frequency, attaining a constant value [15]. On the other hand, the dielectric constant increases with an increase in temperature. It may be due to the increase in space charge polarization at the grain boundary interfaces. Also the low value of dielectric loss at higher frequencies discloses the good optical quality of the sample with low number of defects, which makes the crystal a diserable material for optoelectronic applications [16].

\subsection{DC conductivity studies}

The DC electrical conductivity study of the HMT crystals was performed on Keithley high re-

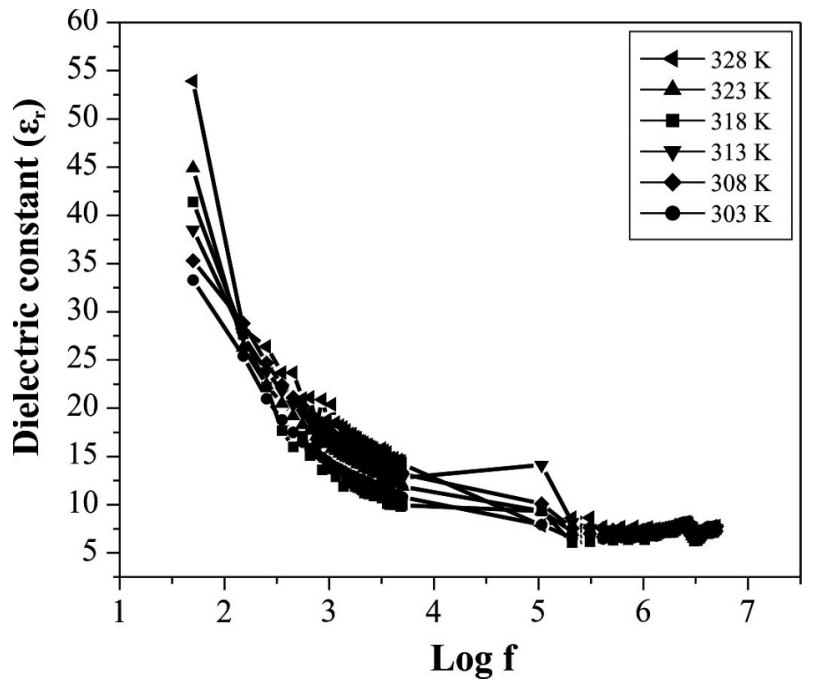

Fig. 3. Dielectric constant vs. frequency.

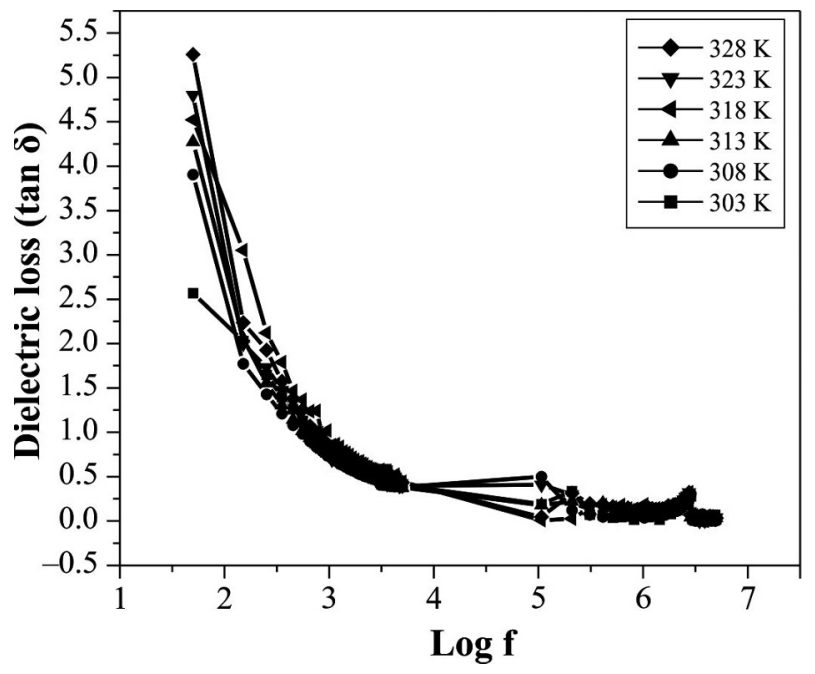

Fig. 4. Dielectric loss vs. frequency.

sistance meter/electrometer $6517 \mathrm{~B}$. In this study the current was measured with respect to the applied voltage across the sample at different temperatures. The sample was sandwiched between two copper electrodes and annealed in an oven. The temperature was controlled by a controller. The current increased linearly with the applied voltage at different temperatures which is shown in Fig. 5 and Fig. 6. The DC electrical conductivity has been calculated using the following formula [17]:

$$
\sigma=\left(\frac{(I \times L)}{(V \times A)}\right)
$$




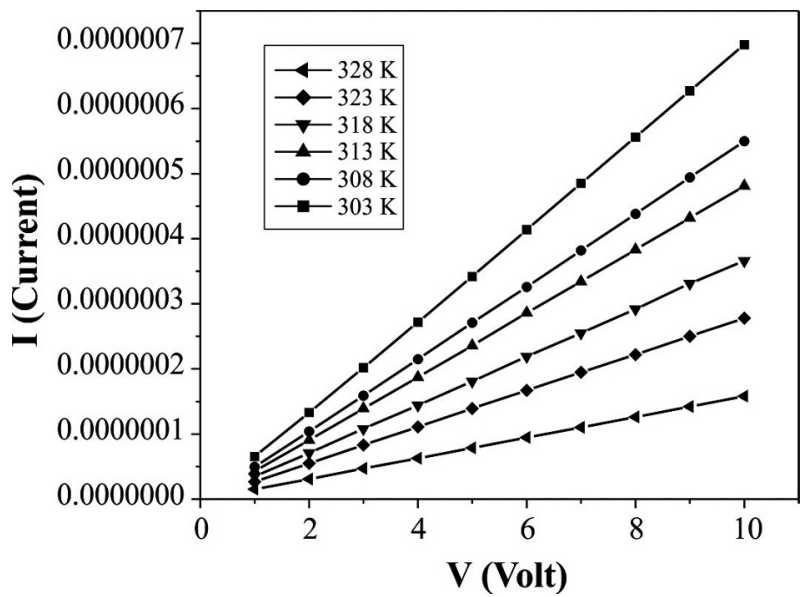

Fig. 5. Current vs. voltage characteristics for HMT crystal.

where $\mathrm{I}$ is the current, $\mathrm{V}$ is the voltage, $\mathrm{L}$ is the thickness of the sample, and A is the cross sectional area of the sample. The DC conductivity increased with an increase in temperature. Fig. 7 depicts the conductivity of the HMT crystal vs. temperature. The plots of $\ln (\sigma)$ vs. 1000/T $(1 / \mathrm{K})$ were found to be very nearly linear (Fig. 8). So, the conductivity values were fitted with Arrhenius expression and the activation energy $\left(\mathrm{E}_{d c}\right)$ has been calculated $[18,19]$ :

$$
\sigma_{d c}=\sigma_{0} \exp \left[\frac{-E_{d c}}{k T}\right]
$$

where, $\sigma_{0}$ is a constant depending on the material, $\mathrm{k}$ is the Boltzmann constant $\left(1.38 \times 10^{-23} \mathrm{JK}^{-1}\right)$, $\mathrm{T}$ is the absolute temperature and the calculated activation energy is $1.36 \mathrm{eV}$.

\subsection{Microhardness}

Microhardness is a measure of bond strength apart from bulk strength. For the device fabrication, good quality crystals with good optical and mechanical properties are needed. The microhardness studies were carried out to determine the mechanical strength of the grown crystal using Leitz Weitzler hardness tester fitted with a diamond pyramidal indenter. The selected flat surfaces of the grown crystals were subjected to a study at room temperature. The time of indentation was $10 \mathrm{~s}$ for all trials. The diagonal lengths of the indented im-

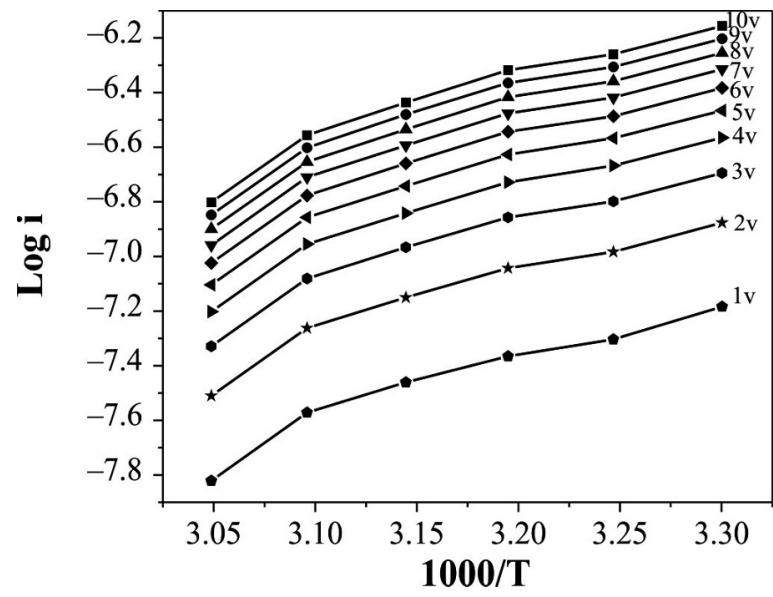

Fig. 6. Log I vs. 1000/T.

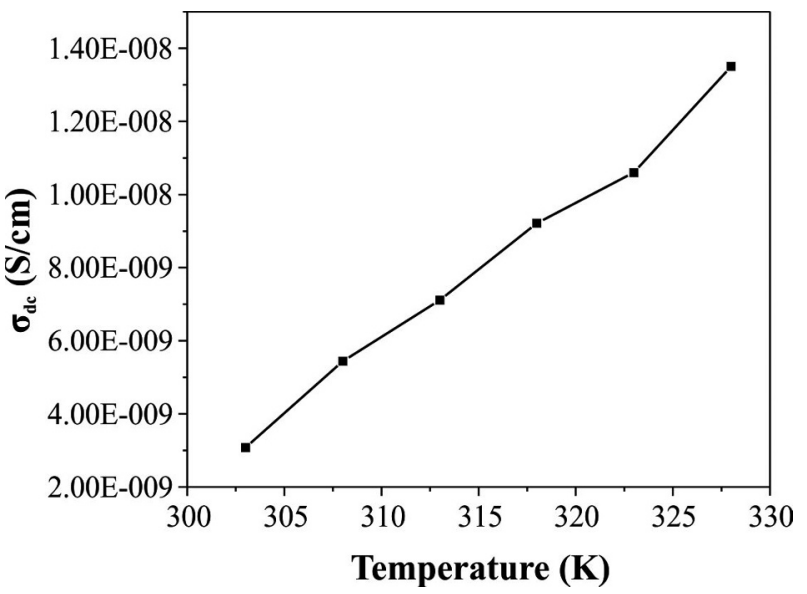

Fig. 7. DC conductivity vs. temperature.

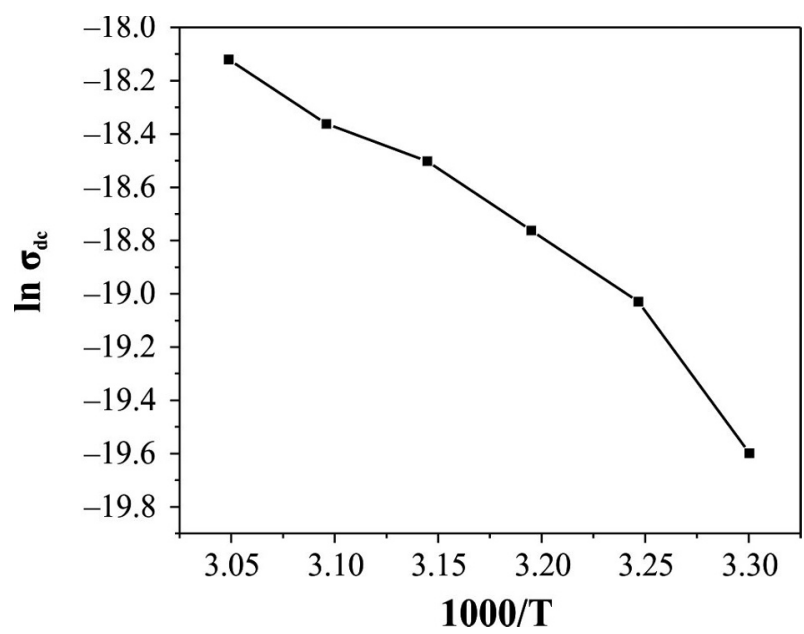

Fig. 8. In $\sigma_{d c}$ vs. reciprocal of absolute temperature $1000 / \mathrm{T}$. 
pression were measured for various loads. The indentation mark was limited to $100 \mathrm{~g}$. The Vickers' microhardness number was calculated using the formula $\mathrm{H}_{v}=1.854 \mathrm{P} / \mathrm{d}^{2}\left(\mathrm{~kg} / \mathrm{mm}^{2}\right)$, where, $\mathrm{P}$ is the applied load in $\mathrm{kg}$ and $\mathrm{d}$ is the diagonal length of the indent in mm. For the applied load above $100 \mathrm{~g}$, microcracks were observed around the impression. It is observed from Fig. 9 that when the load increases, the hardness also increases. According to Onitsch [20] and Hannman [21] ' $v$ ' should lie between 1 and 1.6 for hard materials and is above 1.6 for the soft ones. The plot of $\log \mathrm{P}$ versus $\log \mathrm{d}$ for the hexamethylenetetramine crystal is shown in Fig. 10. The slope of the log P versus log $\mathrm{d}$ curve gives the values of $v$ equal to 1.98 . The resistance pressure is defined as a minimum level of indentation load (W) below which no plastic deformation occurs [22]. Hays and Kendall explained the relationship between indentation test load and indentation size by modifying Kick's law [23]:

$$
\begin{gathered}
P-W=k_{2} d^{2} \\
P=k_{1} d^{n}
\end{gathered}
$$

where $\mathrm{k}_{2}$ is a constant and $(\mathrm{P}-\mathrm{W})$ is the effective indentation test load. Combining equation 4 and 5 we get:

$$
W=k_{1} d^{n}-k_{2} d^{2}
$$

or

$$
d^{n}=W / k_{1}+\left(k_{2} / k_{1}\right) d^{2}
$$

Fig. 11 shows that the plot of $\mathrm{d}^{n}$ vs. $\mathrm{d}^{2}$ is a straight line having a slope $\left(\mathrm{k}_{2} / \mathrm{k}_{1}\right)$ and intercept $\mathrm{W} / \mathrm{k}_{1}$. From these values, we have obtained the value of $\mathrm{W}$ as $0.65 \pm 0.1 \mathrm{~g}$. The plot of $\log (\mathrm{P}-\mathrm{W})$ against $\log \mathrm{d}$, which is depicted in Fig. 12, gives the slope value of 2.02, thus confirming the validity of Hays and Kendall's law. If $\mathrm{n}<2$, the yield strength $\left(\sigma_{y}\right)$ can be calculated using the given equation [24]. Fig. 13 shows the load vs. yield strength of the grown crystal:

$$
\sigma_{y}=H_{v} / 3
$$

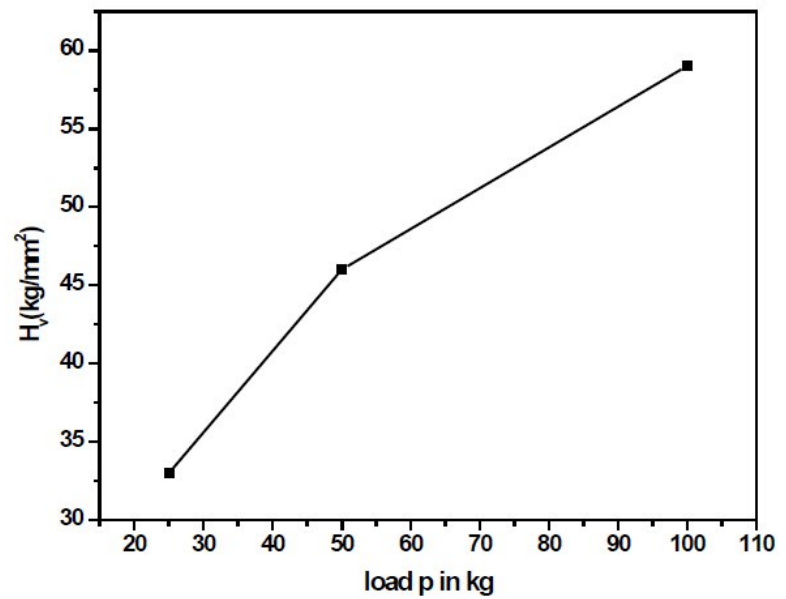

Fig. 9. Hardness vs. applied load.

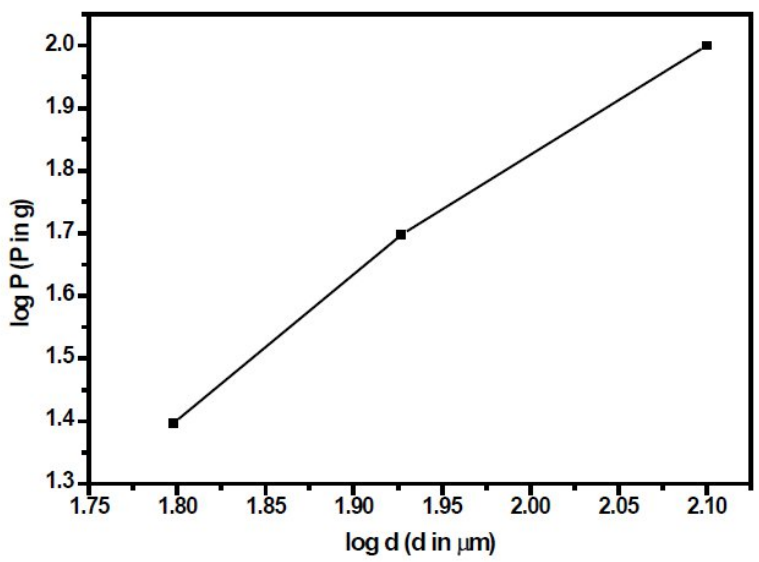

Fig. 10. Log P vs. $\log d$.

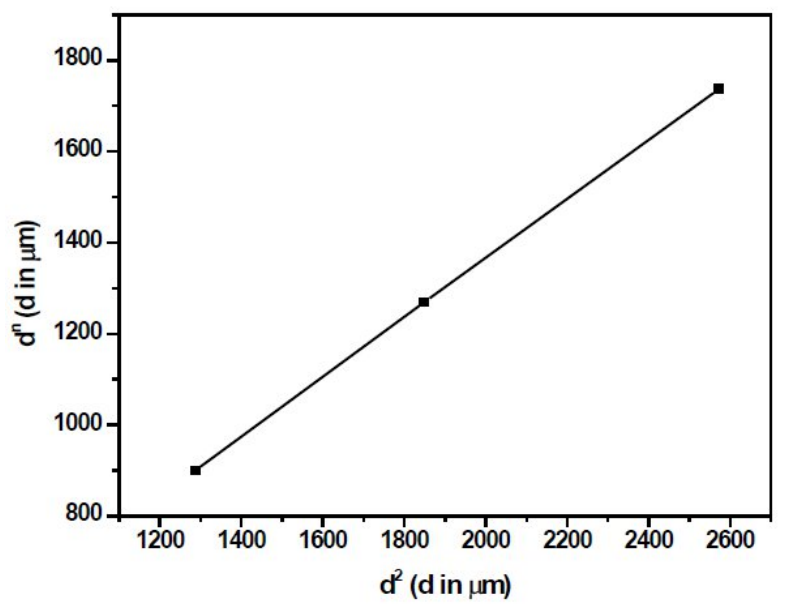

Fig. 11. $d^{n}$ vs. $d^{2}$. 


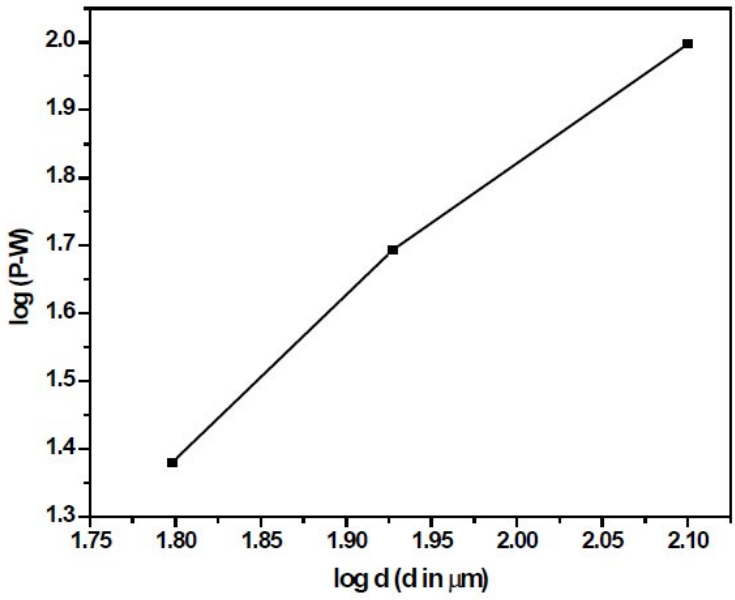

Fig. 12. $\log (\mathrm{P}-\mathrm{W})$ vs. $\log \mathrm{d}$.

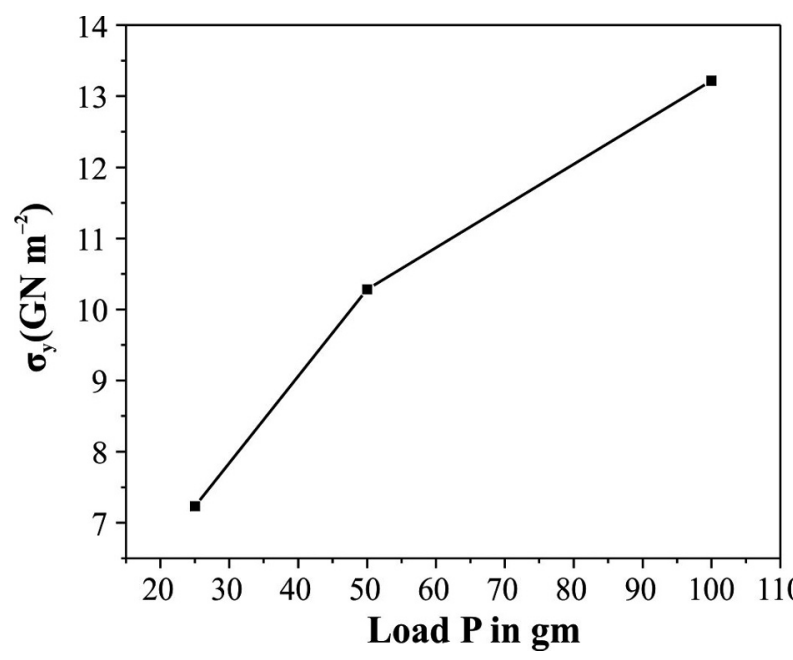

Fig. 13. $\sigma_{y}$ vs. load P.

\subsection{Photoconductivity studies}

The photoconductivity study of the HMT crystals was performed on Keithley high resistance meter/electrometer $6517 \mathrm{~B}$. Thin copper wires were fixed on the opposite surfaces of the crystal using good quality silver paste in order to get good ohmic contact. To measure the photocurrent the sample was exposed to the radiation from the $100 \mathrm{~W}$ halogen lamp containing iodine vapour and tungsten filament. The photocurrent and the dark current were recorded as a function of applied electric field, Fig. 14. The graph shows that the photocurrent is higher than the dark current, which is known as positive photoconductivity [25]. This type of photoconductivity is due to the increase in the number

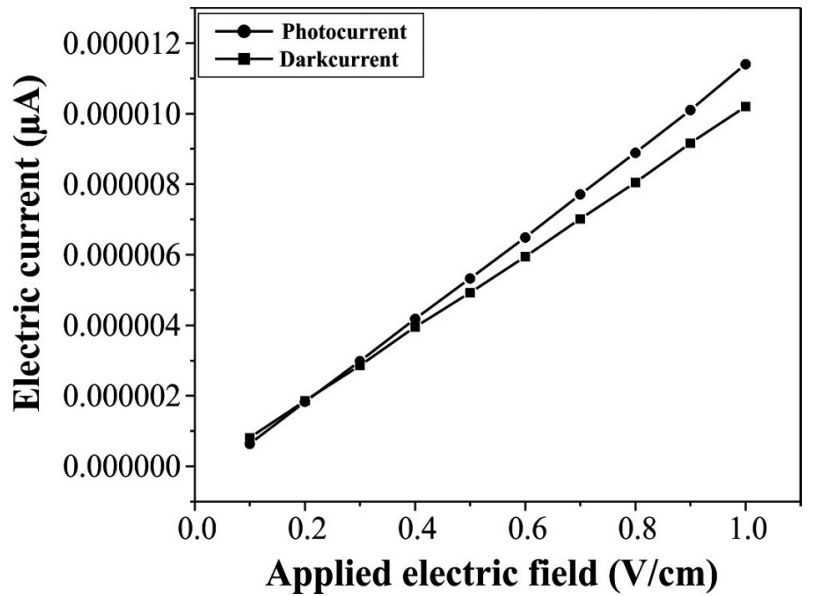

Fig. 14. Variation of photocurrent and dark current with applied electric field.

of charge carriers or their lifetime. As the crystal acquiring high band gap for photon absorption, the applications are extended towards soliton wave communication [26].

\subsection{Powder SHG}

The powder SHG test is a valuable method for initial screening of NLO materials [27]. The crystals were grinded into a powder that was densely packed in a capillary tube $[1 \mu]$. Q-switched $\mathrm{Nd}$ :YAG laser was used to investigate the SHG of the grown crystals. A fundamental laser beam of $1064 \mathrm{~nm}$ was directed on the powder sample. The input power of the incident beam, measured with a power meter, was 3 MJ. The SHG behavior of the material was confirmed by the emission of green radiation $(\lambda=532 \mathrm{~nm})$. This green emission was finally detected by a photo multiplier tube and displayed on a storage oscilloscope. KDP crystals were powdered and used as a reference material in the SHG measurement. The relative efficiency of HMT is comparable with the KDP. The SHG signal outputs are $13 \mathrm{mV}$ and $26 \mathrm{mV}$ for KDP and HMT, respectively. It is two times greater than the value for KDP (Table 2).

\section{Conclusions}

Optically good quality single crystals of hexamethylenetetramine with the size of 
Table 2. Comparison of SHG signals output.

\begin{tabular}{ccc}
\hline $\begin{array}{c}\text { Input power } \\
(\mathrm{mJ} / \text { pulse })\end{array}$ & $\begin{array}{cc}(\mathrm{mV}) & (\mathrm{mV})\end{array}$ \\
\hline \hline 3 & 13 & 26 \\
\hline
\end{tabular}

$\left(10 \times 10 \times 5 \mathrm{~mm}^{3}\right)$ were successfully grown by slow evaporation solution growth technique at a constant temperature $\left(35^{\circ} \mathrm{C}\right)$. Single crystal XRD confirmed that the crystal belongs to the cubic system. Powder X-ray diffraction analysis verified the crystalline nature of the crystal. The optical transmittance in the entire visible region makes the crystal a potential candidate for optoelectronic applications. The photoconductivity study revealed the positive photoconductivity nature. Powder second harmonic generation efficiency of the grown crystal was confirmed by the green light emission.

\section{Acknowledgements}

The authors gratefully acknowledge the financial support from the DST, Government of India, for the major research project (SB/EMEQ-293/2013). One of the authors, B. Babu thanks UGC Networking Resource Centre, School of Chemistry, University of Hyderabad, India for awarding Visiting Research Fellowship.

\section{References}

[1] Angeli Mary P.A., Dhanuskodi S., Cryst. Res. Technol., 36 (2001), 1231.

[2] Vesta C., Uthrakumar R., Babu Varghese, $J$. Cryst. Growth., 311 (2009), 1516.

[3] Swets D.E., Jorgensen E.S., J. Cryst. Growth., 5 (1969), 299.

[4] Duckett R.A., Lang A.R., J. Cryst. Growth., 18 (1973), 135.

[5] Heilmeier G.H., Appl. Optics, 3 (1964), 1281.

[6] LEE R.W., Appl. Optics, 8 (1969), 1385.

[7] Chen L., Huang F., Li S., Shen Y., Xie A., Pan J., Zhang Y., CAI Y., J. Solid State Chem., 184 (2011), 2825 .
[8] Singh G., BARAnWAL B.P., KaPOOR I.P.S., KUMAR D., Fröhlich R., J. Phys. Chem. A, 111 (2007), 12972.

[9] Kampermann S.P., Ruble J.R., Craven B.M., Acta Crystallogr. B, 50 (1994), 737.

[10] Chen L.-J., Chuang Y.-J., Mater. Lett., 68 (2012), 460.

[11] Yi W.-B., CAI C., J. Hazard. Mater, 150 (2008), 839.

[12] Becka L.N., Cruickshank D.W.J., P. Roy. Soc. Lond. A Mat., (1963), 273.

[13] Chandrasekaran J., BabU B., BalaprabhaKaran S., Ilayabarathi P., MAADESWARAN P., J. Optoelectron. Adv. M., 6 (2012), 211.

[14] Chandrasekaran J., Ilayabharathi P., MaAdeswaran P., Mohamed Kutty P., Pari S., Opt. Commun., 285 (2012), 2096.

[15] Lenin M., Balamurugan N., Ramasamy P., Cryst. Res. Technol., 42 (2007), 39.

[16] Dhanaraj P.V., RaJesh N.P., Physica B, 406 (2011), 12.

[17] BABU B., Chandrasekaran J., BALAPRABhakaran S., Ilyabarathi P., Mater. Sci.-Poland., 31 (2013) 151.

[18] Priya M., Mahadevan C.K., Cryst. Res. Technol., 44 (2009), 92.

[19] Mariappan R., Ponnuswamy V., Ragavendar M., Mat. Sci. Semicon. Proc., 15 (2012), 199.

[20] Onitsch E.M., Mikroscopia, 2 (1947), 131.

[21] Hannman M., Metall. Manch., 23 (1941), 135.

[22] Kumari P.N.S., Kalainathan S., RaJ N.A.N., Mater. Lett., 62 (2008), 305.

[23] Hays C., Kendall E.G., Metallography, 6 (1973), 275.

[24] Cahoon J.P., Broughton W.H., Katzuk A.R., Metall. Trans., 2 (1971), 1979.

[25] Chandrasekaran J., Ilayabarathi P., MaAdeswaran P., Opt. Commun., 285 (2012), 3872.

[26] Boaz B.M., Priya S.M.N., Linet J.M., Prasath P.M.D., DAs S.J., Opt. Mater., 29 (2007), 827.

[27] Kurtz S.K., Perry T.T., J. Appl. Phys., 39 (1968), 3798.
Received 2013-02-01

Accepted 2014-03-03 